\title{
L'emploi des jeux dans l'enseignement des langues étrangères : Du traditionnel au numérique
}

\section{Laurence Schmoll}

\section{(2) OpenEdition}

\section{Journals}

Édition électronique

URL : http://journals.openedition.org/sdj/628

DOI : $10.4000 /$ sdj. 628

ISSN : 2269-2657

Éditeur

Laboratoire EXPERICE - Centre de Recherche Interuniversitaire Expérience Ressources Culturelles Education

\section{Référence électronique}

Laurence Schmoll, «L'emploi des jeux dans l'enseignement des langues étrangères : Du traditionnel au numérique », Sciences du jeu [En ligne], 5 | 2016, mis en ligne le 28 février 2016, consulté le 21 décembre 2020. URL : http://journals.openedition.org/sdj/628; DOI : https://doi.org/10.4000/sdj.628

Ce document a été généré automatiquement le 21 décembre 2020.

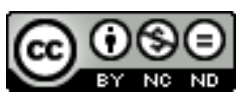

La revue Sciences du jeu est mise à disposition selon les termes de la Licence Creative Commons Attribution - Pas d'Utilisation Commerciale - Pas de Modification 4.0 International. 


\title{
L'emploi des jeux dans l'enseignement des langues étrangères : Du traditionnel au numérique
}

\author{
Laurence Schmoll
}

1 Que ce soit sous sa forme traditionnelle ou sa forme numérique, le jeu tient une place peu définie dans la classe de langue. Il recouvre des emplois divers en fonction du niveau et de la catégorie d'âge des apprenants et suscite des réactions divergentes chez les apprenants et chez les enseignants. S'il existe des publications qui s'intéressent à la question du jeu dans l'enseignement des langues, celles-ci sont bien souvent à l'origine de réflexions et de mises en pratique ponctuelles, quand elles ne sont pas complètement empiriques (Silva, 2008). L'introduction des Technologies de l'Information et de la Communication (TIC), dans l'enseignement depuis les années 1990, a par ailleurs laissé la porte entrebâillée aux jeux numériques et, pourrions-nous le supposer, à de nouveaux usages.

2 L'objet de cet article est de proposer un panorama de l'utilisation des jeux dans l'enseignement des langues, et plus particulièrement du Français Langue Étrangère (FLE) à destination des "grands adolescents $»^{1}$ et des adultes, en observant comment s'est effectuée la transition du jeu traditionnel au jeu numérique.

3 À cette fin, nous ne considérerons pas le jeu selon une définition précise, l'élément essentiel selon nous étant "que l'on joue pour le plaisir procuré par l'activité " (Brougère, 2012, p. 118). Tout le travail du concepteur ou de l'enseignant - créateur ou utilisateur - consiste donc à faire en sorte que le jeu proposé soit le plus susceptible possible de placer l'apprenant dans une « attitude ludique » (Henriot, 1983, p. 83; Genvo et Poix, 2003, p. 78). Nous retiendrons, par conséquent, les deux grandes caractéristiques présentées par Brougère (2012, pp.122-124) qui font du jeu une activité de «second degré " suffisamment ouverte pour que le joueur puisse l'investir en prenant des "décisions" (ne serait-ce qu'en continuant ou arrêtant de jouer). De ces deux caractéristiques découlent trois autres critères: les décisions sont corrélées à des 
« règles »; elles sont guidées par un enjeu dont l'issue est « incertaine »; le second degré permet cette «frivolité » que la réalité n'autorise pas toujours en mettant à distance les conséquences des décisions prises (Brougère, 2012). Ces cinq éléments permettent d'orienter la réflexion, sans toutefois la figer, sur ce qui est susceptible d'être qualifié de jeu ou de ludique, dans le sens que nous pouvons trouver dans le dictionnaire, à savoir « relatif au jeu » (Rey, 2012, p. 1487).

Gardant à l'esprit ce positionnement, il nous semble utile de considérer quelles relations les acteurs de l'enseignement des langues observent avec le jeu, traditionnel tout d'abord, numérique ensuite, à travers une analyse des supports et activités présentés comme tel. Nous nous demanderons notamment si le passage au numérique a permis de préserver, pour reprendre les termes de la définition du serious game d'Alvarez (2007, p. 35), les « ressorts ludiques » spécifiques à ces jeux ou les a modifiés au profit de l'« intention » et du contenu sérieux. Nous verrons quelle peut être la valeur ajoutée du jeu numérique par rapport au jeu traditionnel à la lumière des méthodologies d'enseignement-apprentissage actuelles et comment, pour aller plus loin, penser la conception d'un jeu vidéo éducatif en respectant, d'une part, un certain équilibre entre ludique et sérieux et d'autre part, la démarche didactique de départ. Pour ce faire, nous prendrons pour exemple le cheminement par lequel nous sommes passée pour concevoir un jeu vidéo sérieux en immersion 3D stéréoscopique pour l'apprentissage du français et de l'allemand: Architecte 2015.

\section{Jeu traditionnel et enseignement des langues}

5 L'idée d'introduire le jeu dans l'éducation, et par extension dans l'enseignement des langues, fait sa première apparition de façon périphérique au début du $18^{\mathrm{e}}$ siècle, non pas dans l'enseignement scolaire français, mais chez quelques précepteurs qui proposent en vain de réformer le système traditionnel. Puren (2012 [1988], p. 29) fait ainsi référence à De Vallange (1730) et son Art d'enségner le latin aux petits enfans en les divertissant et sans qu'ils s'en aperçoivent; dépendance de l'Art d'élever la jeunesse selon la différence des âges, du sexe et des conditions qui propose entre autres d'employer des jeux de cartes pour l'apprentissage de la lecture et de la grammaire latines et préconise de manière générale d'associer l'étude et le jeu, ce dernier passant par un support (éventails, poupées) ou une activité ludiques (comme la " grammaire digitale » où l'on apprend « en badinant sur les doigts »).

Le jeu ne connaît cependant pas de fortune particulière dans les méthodologies anciennes, et encore moins dans la formation des adultes, jusqu'aux cours audiovisuels de troisième génération dans les années 1980 et l'approche communicative. C'est à ce moment que sont abandonnées progressivement les théories de référence structuraliste et béhavioriste qui, dans les années 1950-1960, avaient orienté l'enseignement de la langue sur l'apprentissage des formes et des structures. Le constructivisme et le cognitivisme replacent alors l'apprenant au centre d'un apprentissage en contexte ${ }^{2}$. À partir de ce moment-là, l'enseignement des langues intègre des activités « plus variées et plus «créatrices", comme les jeux, les simulations et les jeux de rôles" (Puren, 2012 [1988], p. 258). L'ouvrage de Caré et Debyser (1978), intitulé Jeu, langage et créativité. Les jeux dans la classe de français, est représentatif de ce nouvel intérêt à tel point que les termes «ludique » et «jeu» font de plus en plus leur apparition dans les programmes officiels, les référentiels et les différents supports d'apprentissage. 
7 Toutefois, employer une rhétorique du jeu pour désigner un objet ou une activité n'est pas suffisant pour leur apporter le caractère qu'elle désigne. Il semble opportun de se pencher sur l'utilisation qui en est faite dans des documents qui se veulent des outils de référence pour l'apprentissage des langues. Ainsi, avant même d'entrer dans une description et une analyse de l'emploi des jeux en classe de langue, nous nous demandons ce que les auteurs de ces ouvrages entendent par ces deux termes - «jeu», «ludique »appliqués à l'enseignement.

8 Silva (2008, p. 21) souligne que le Cadre européen commun de référence pour les langues, texte de 196 pages qui a, entre autres, pour ambition d'harmoniser les pratiques d'enseignement et d'évaluation dans le domaine des langues, n'accorde qu'un chapitre d'une quinzaine de lignes à la question de l'« utilisation ludique de la langue » (Conseil de l'Europe, 2001, p. 47). Ce dernier se présente essentiellement sous la forme d'une liste de jeux portant sur le lexique : les lettres (le pendu, Scrabble), les mots (anagrammes, rébus) ou encore les jeux de mots (au sens de calembours et autres contrepèteries). Il est fait référence au jeu à deux autres reprises. L'une présente le jeu comme une visée d'apprentissage, par exemple « dire à des enfants que l'activité qu'on leur propose leur permettra de jouer ensuite, dans la langue étrangère, au jeu des Sept Familles » (Conseil de l'Europe, 2001, p. 107). L'autre cite les «jeux coopératifs et compétitifs» (Conseil de l'Europe, 2001, p. 112), parmi d'autres activités possibles pour un travail de groupe au moyen de supports dits techniques (ordinateurs, etc.).

Peu présent, le jeu est donc désigné avant tout comme un support d'apprentissage et non comme un moyen et le texte ne fait jamais référence ni à ses apports potentiels, ni à ses caractéristiques. Le Cadre met en relation le jeu comme visée d'apprentissage et la motivation qui peut en être la conséquence. Les notions de coopération et de compétition sont également évoquées sans plus d'approfondissement et uniquement en rapport avec les TIC.

10 Pour aller plus loin, nous avons recherché la présence de ces deux termes dans dix manuels de langue pour l'enseignement du Français Langue Étrangère. Nous nous sommes surtout attachée à collecter les mots « jeu » et « ludique » dans les avant-propos du manuel de l'apprenant et dans les guides pédagogiques à destination des enseignants. Un seul des dix manuels utilise explicitement les jeux. L'ambition affichée est de réemployer le «lexique dans un contexte amusant » (Baglieto, Girardeau \& Mistichelli, 2011a, p. 6). Ces jeux portent sur la coopération (transmettre un message à son voisin qui doit le restituer correctement) ou la compétition (formation de groupe et comptage de points, mémorisation et rapidité). Certains sont des jeux de langage, d'autres des jeux de plateau (comme le jeu de l'oie) ou encore des devinettes. La plupart porte sur le vocabulaire ou la grammaire, plus rarement sur un aspect culturel (voir Figures 1 et 2). 
Figure 1

1

8 meubles ou objets de la maison sont cachés.

Retrouvez les mots.

Attention, c'est vertical $\uparrow$, horizontal $\rightarrow$ ou diagonal $₫$ !

\begin{tabular}{|c|c|c|c|c|c|c|c|c|c|c|c|c|}
\hline B & D & $\varepsilon$ & $A$ & $\mathrm{~L}$ & $\mathrm{P}$ & M & $\mathrm{K}$ & $\mathrm{T}$ & 1 & 0 & 8 & A \\
\hline R & E & $f$ & $R$ & 1 & 6 & $E$ & R & A & 1 & $\mathbf{E}$ & U & R \\
\hline L & $\mathrm{M}$ & A & $s$ & $T$ & c & $c$ & $c$ & $\mathrm{~s}$ & H & I & E & $M$ \\
\hline 0 & 1 & U & $\mathbf{N}$ & c & H & Q & M & L & B & $M$ & A & 0 \\
\hline$H$ & $P$ & $T$ & 0 & L & A & $\mathrm{M}$ & p & $\varepsilon$ & $\pi$ & s & T & 1 \\
\hline 1 & 1 & $\mathrm{E}$ & L. & M & 1 & $N$ & C & $A$ & U & $c$ & D & R \\
\hline$u$ & $v$ & U & $x$ & $\gamma$ & 5 & E & A & $F$ & G & D & и & E \\
\hline 1 & E & 1 & $\mathrm{~N}$ & $p$ & $\mathbf{E}$ & 5 & v & $p$ & B & $E$ & t. & a \\
\hline L & M & L & 0 & $\mathrm{P}$ & 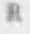 & $\mathrm{T}$ & V & A & E & c & F & $\varepsilon$ \\
\hline
\end{tabular}

Mots mêlés sur le vocabulaire de la maison (Baglieto, Girardeau, Mistichelli, 2011b, p. 76)

Figure 2

JOUR 7 RENDEZ-VOUS1 - À SAVOIR, À PRONONCER, ACTIVITÉ 3 p. 120

Jeu de l'histoire.

Nombre de joueurs: groupes de 5 ou 6 apprenants

Règle

Un joueur commence une histoire avec une phrase au passé composé.

Un autre joueur continue l'histoire avec une autre phrase au passé composé et ainsi de suite.

Le joueur qui se trompe d"auxiliaire au passé composé est éliminé

Le groupe qui a produit le plus de phrases a gagné.

Jeu mêlant coopération et compétition portant sur le passé composé (Baglieto, Girardeau, Mistichelli, 2011a, p. 202)

11 Cinq manuels emploient le mot « ludique » (Abry et alii, 2007a, p. 7 ; Capelle \& Menand, 2009b, p. 6 ; Dubois, Lerolle, 2008, p. 6 ; Hugot et alii, 2012, p. 3 et 4 ; Monnerie-Goarin et alii, 2006b, p. 8 et 11). Ce dernier renvoie à des activités qui ne sont pas présentées comme des jeux, mais qui sont plus ouvertes que les exercices traditionnels et dont le but est d'inciter à l'échange. Il en est ainsi des jeux de rôles, des tâches et des projets. Le terme est alors accompagné d'autres mots-clés, tels que "créatif», "motivant», « investissement », « libre », " récréatif », « valorisant ». Un seul ouvrage sur les cinq 
(Capelle, Menand, 2009b) emploie le terme « ludique " pour faire référence à des activités numériques portant sur la grammaire ou le vocabulaire. Faut-il comprendre que le caractère interactif puisse donner d'emblée une couleur plus attrayante à ce type d'activités?

Trois autres manuels (Flumian \& Catherine et alii, 2011 ; Mérieux \& Loiseau, 2004a, 2008) ne font aucune référence au jeu ou au ludique, mais présentent également les activités ouvertes du type «jeux de rôles » comme un facteur de motivation, en insistant sur la notion de plaisir dans l'apprentissage. Enfin, un seul manuel tient un discours où le ludique ne semble pas avoir sa place. Lorsqu'il est question des jeux de rôles, ce dernier précise : » on abordera ces jeux de rôles non pas comme des moments de divertissement (encore qu'ils puissent l'être), mais comme les étapes obligées d'une formation » (Girardet \& Gibbe, 2010a, p. VII).

Pour conclure cette rapide analyse, sont qualifiées de «ludiques » par les concepteurs de manuel les activités ouvertes, propices à l'interaction entre les apprenants et dans une moindre mesure, les activités proposées sur support multimédia. Nous constatons qu'une confusion est faite entre le jeu et le jeu de rôles, du fait notamment de la proximité des termes, mais également pour la raison que tous deux partagent la caractéristique de second degré et d'absence de conséquence sur la réalité. Un jeu de rôles en éducation correspond davantage à une simulation durant laquelle les apprenants incarnent un personnage dans un contexte donné de la vie réelle et en improvisant (plus ou moins en fonction du temps de préparation) un dialogue. Cette activité peut difficilement, à notre sens, être qualifiée de jeu (à moins que les apprenants l'investissent comme tel), car elle ne présente pas de règles (mais des consignes) et son enjeu n'intègre pas la notion de victoire ou de récompense bien que nous puissions parler de gain à l'apprentissage. Pour appuyer notre propos, citons Sauvé, Renaud et Kaufman qui proposent de distinguer jeu et simulation éducatifs de la façon suivante :

Le jeu est une situation fictive, fantaisiste ou artificielle dans laquelle des joueurs, mis en position de conflit [...], sont soumis à des règles qui structurent leurs actions en vue d'atteindre des objectifs d'apprentissage et un but déterminé par le jeu [...]. $\mathrm{Au}$ contraire, la simulation se veut un modèle simplifié, dynamique et juste d'une réalité définie comme un système. [...] La simulation n'implique pas nécessairement un conflit, une compétition, et la personne qui l'utilise ne cherche pas à gagner, ce qui est le cas dans le jeu (Renaud et Kaufman, 2010, p. 35).

Si le jeu de rôles ou la tâche proposés par l'enseignant peuvent générer de la motivation chez les apprenants, nous supposons que c'est par leur caractère plus ouvert, les apprenants y expérimentant une marge de liberté plus grande qu'avec des exercices traditionnels. Il nous paraît moins évident de les qualifier d'emblée de «ludique » ou d'« amusant » et dès lors, l'utilisation de ce mot revêt un caractère plutôt commercial.

En mettant de côté les jeux de rôles et les projets, le jeu en classe de langue semble alors se concentrer sur les jeux de société familiers, les jeux de lettres et les devinettes portant sur un savoir lexical ou grammatical, éventuellement culturel. De façon plus empirique, d'autres activités effectuées en classe pourraient au minimum être susceptibles de provoquer une attitude ludique chez les apprenants, tout en s'ouvrant aux interactions entre les utilisateurs et à un réinvestissement plus important en compréhension et en production. Il s'agit d'activités qui placent les apprenants en position de compétition ou de coopération et qui emploient les mécanismes du jeu, procédé réactualisé sous le nom de gamification (Deterding et al., 2011). Elles sont souvent empruntées au domaine de l'enfance et mettent pour la plupart le corps en action. Il existe ainsi de nombreuses 
adaptations de "Jacques a dit» ou du jeu de cache-cache, dont nous pouvons trouver quelques exemples dans les travaux de Weiss (2002) ou ceux de Silva (2008). Les activités qui font appel à la créativité, notamment narrative, à la mémoire ou au hasard pourraient également entrer dans cette catégorie. Toutefois, les jeux de société et de lettres restent ceux qui jouissent plus particulièrement d'une bonne fortune auprès des enseignants qui ont tendance à les utiliser comme un intermède ludique qui ouvre, ponctue ou clôt le cours.

\section{Le numérique en classe de langue : quid du jeu?}

L'arrivée des TICE, et notamment d'Internet, en classe de langue apporte avec elle l'espoir d'un support complémentaire propice aux interactions écrites et orales. Nous avions constaté il y a quelques années (Schmoll \& Koecher, 2006, p. 590) que les spécificités d'Internet étaient très peu exploitées. D'une part, l'interactivité se cantonnait à remplir des cases (exercices lacunaires) ou sélectionner des items (QCM) pour un feedback pas toujours présent et, quand c'était le cas, qui se réduisait souvent à une correction sans plus d'explications. D'autre part, les interactions possibles entre apprenants ou même entre usagers étaient quasi inexistantes.

Quelques années plus tard sont apparus des sites pédagogiques exploitant les possibilités des forums et des blogs pour amener les apprenants non seulement à communiquer entre eux, mais surtout à interagir avec des locuteurs natifs. À travers cette pratique a émergé la notion de "didactique invisible", développée par Ollivier (2012). Ce dernier constate que les productions des apprenants réalisées dans des conditions de communication proches de la vie réelle, mais qui ont lieu dans une situation de communication simulée, sont fortement influencées par le contexte de la classe et la présence de l'enseignant. Au final, le destinataire n'est pas l'interlocuteur imaginaire décidé par l'enseignant, mais reste bien l'enseignant lui-même, puisque c'est lui qui écoute ou lit vraiment le message et qui l'évalue. Le projet Babelweb reflète ce concept de didactique invisible, car le but est de proposer aux apprenants des espaces de publication dédiés sur lesquels apprenants et utilisateurs peuvent interagir hors des limites de la classe et sans que cet aspect apprentissage soit explicitement affiché.

Dans le même esprit, utiliser des jeux numériques à des fins pédagogiques devrait permettre de sortir du contexte de la classe. L'apprenant change de statut en devenant joueur et il est possible, au moins en partie, de lui faire oublier le contenu sérieux s'il se laisse prendre par les ressorts ludiques et se place par conséquent dans une attitude ludique. Les apports supposés sont nombreux et présentés de manière plus empirique que scientifique. Le principal avantage avancé est de susciter la motivation de l'apprenant et de déstigmatiser la prise de risque dans la langue-cible, ainsi que l'évaluation, car s'il y a échec, le joueur-apprenant devrait a priori plutôt considérer qu'il a perdu au jeu et non qu'il a échoué d'un point de vue linguistique. Une enseignante ayant expérimenté les jeux numériques en classe de langue nous a rapporté que certains de ses apprenants recommencent le jeu jusqu'à ce qu'ils aient un score parfait. ${ }^{3}$

Ces considérations sur les jeux numériques sont également valables pour les jeux traditionnels et nous avons observé dans une autre étude (Schmoll, 2011, p. 149) que beaucoup de jeux numériques ayant pour vocation un entraînement au FLE sont en réalité des jeux traditionnels existants détournés sur support informatique. Il existe différents jeux de l'oie, pendus ou encore mots croisés dont le but est de promouvoir un 
entraînement linguistique dans le but de mémoriser du vocabulaire, une conjugaison ou une structure grammaticale (voir Figure 3).

Figure 3

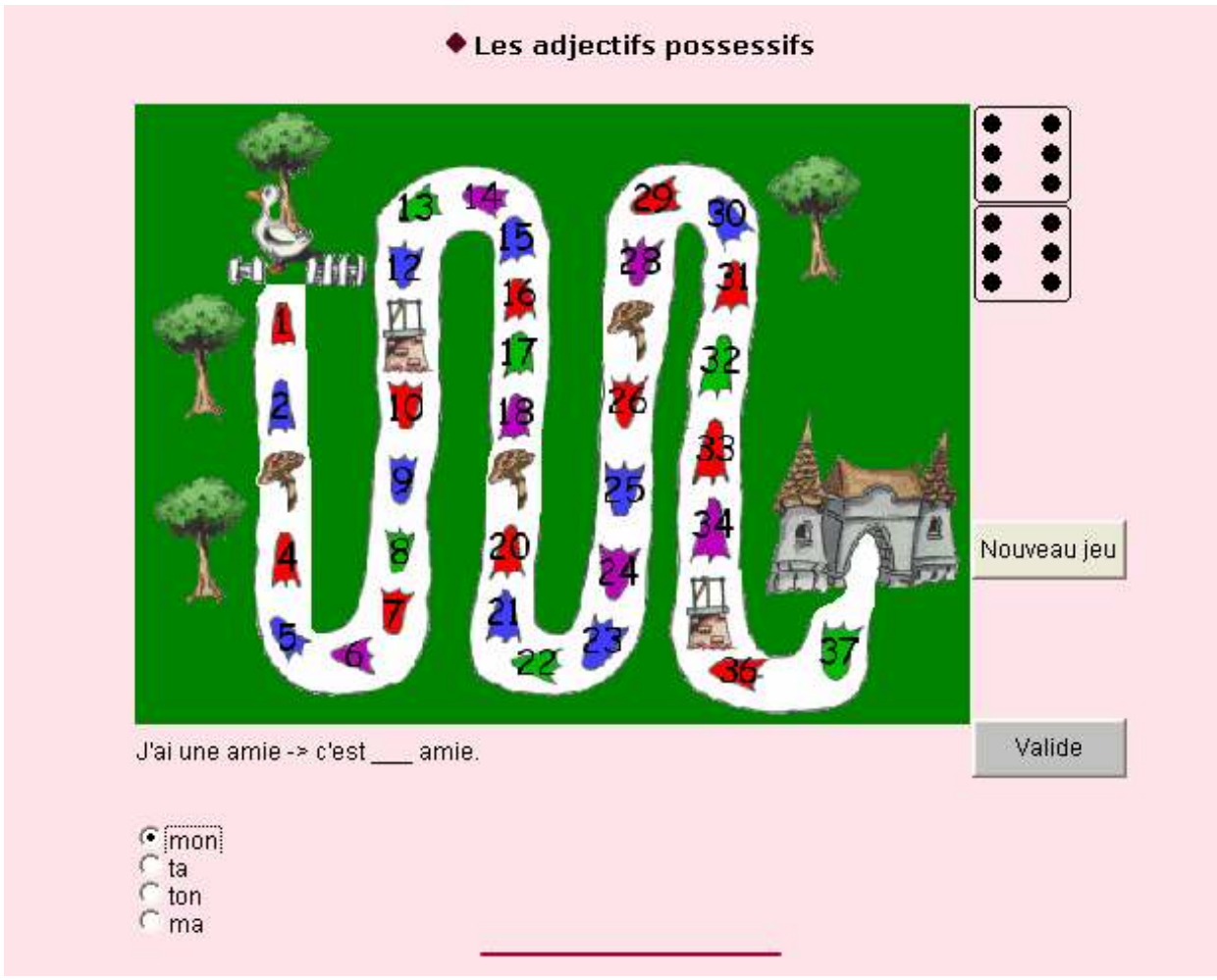

Jeu de l'oie en ligne portant sur les adjectifs possessifs (http://jeudeloie.free.fr/)

Nous avons pu noter par ailleurs que certains exercices fermés du type exercices lacunaires ou QCM revêtent une aura ludique dans le discours des acteurs de l'enseignement, dès lors qu'ils sont présentés sur support multimédia. Par exemple, Le nouveau Taxi 1 présente dans son guide pédagogique des activités autocorrectives sur DVD-Rom en précisant que leur ambition est de « vérifier ses acquis de manière ludique et interactive en grammaire, vocabulaire et conjugaison» (Capelle \& Menand, 2009b, p. 6).

21 Face à ce type de jeux et d'activités, nous nous demandons quelle est la valeur ajoutée du support informatique par rapport aux jeux traditionnels. Nous nous retrouvons dans le même cas de figure que celle des sites pédagogiques cités précédemment qui, dans un premier temps, ne présentaient pas d'originalité particulière par rapport à un support papier et pour lesquels les concepteurs, se reposant sur l'attrait de la nouveauté, se contentaient de reprendre le modèle du manuel de langue avec des séries d'exercices formels. Là aussi, les jeux proposés ne sont en réalité que des exercices fermés cachés derrière un habillage dit ludique, qui font un usage timide des potentialités du multimédia et qui n'incitent pas l'apprenant à s'impliquer. Le même constat peut être appliqué aux logiciels dits ludoéducatifs qui exploitent les mécaniques du jeu vidéo comme le système de récompense, mais peinent souvent à activer une attitude ludique chez l'utilisateur (Mauco, 2012). Ce dernier n'est pas dupe ; les objectifs d'apprentissage paraissent évidents. Ainsi, la didactique invisible n'opère pas vraiment, peut-être même moins qu'avec un jeu traditionnel utilisé dans le cadre du cours de langue. 

numérique, pourrait présenter un atout de taille pour l'enseignement des langues, à condition d'aller plus loin qu'une simple transposition de l'existant. En effet, pour en revenir aux méthodologies d'enseignement des langues, nous nous situons depuis les années 1980 dans une approche communicative et plus récemment dans une perspective actionnelle, prolongement de la première. L'approche communicative a pour principe de considérer l'apprenant comme étant acteur de son apprentissage et a pour ambition de lui donner les moyens d'apprendre à apprendre et de développer une compétence de communication (Hymes, 1984, p. 74). L'apprentissage n'est de ce fait plus centré sur les compétences linguistiques (lexique, grammaire, phonétique), même si elles sont toujours présentes, mais sur la capacité à interagir, en travaillant les compétences de compréhension et de production orales et écrites, à travers des objectifs dits communicatifs, tels que «se présenter» ou «demander poliment». C'est ainsi qu'apparaît le jeu de rôles dont nous avons parlé plus haut, dont le but est d'entraîner les apprenants à communiquer oralement. Dans ce contexte, le web social, avec ses possibilités d'ouverture sur le monde et d'interactions à distance, a suscité un réel intérêt dans le domaine de la didactique des langues. l'apprenant comme un acteur social, autrement dit quelqu'un, non plus seulement qui communique, mais qui agit dans la société et qui, pour ce faire, emploie des stratégies qui peuvent être langagières ou non-langagières. De ce point de vue, l'apprentissage ne va plus seulement être centré sur les compétences langagières (telles que la compréhension et la production), mais également sur toutes les stratégies que l'usager est susceptible de développer pour atteindre son but, qui n'est pas la communication, mais une action, une "tâche " (Ellis, 2003, p. 5), comme " acheter du pain à la boulangerie ", "organiser une fête des voisins ", etc.

Est définie comme tâche toute visée actionnelle que l'acteur se représente comme devant parvenir à un résultat donné en fonction d'un problème à résoudre, d'une obligation à remplir, d'un but qu'on s'est fixé. (Conseil de l'Europe, 2001, p. 16)

Le passage du jeu de rôles à la tâche marque un tournant dans l'enseignement des langues, car, désormais, l'enseignant n'est plus censé construire ses séances en fonction d'objectifs communicatifs, mais doit d'abord décider quelle va être la tâche à effectuer, ainsi que le contexte dans lequel elle va avoir lieu, et en fonction de cette dernière, fournir aux apprenants ce dont ils vont avoir besoin langagièrement et stratégiquement pour la réussir.

De ce point de vue, les jeux vidéo plus ouverts, tels que les jeux d'aventure, qui permettent une réelle participation et des interactions entre utilisateurs ou avec des personnages non-joueurs (PNJ), présentent un intérêt majeur pour l'enseignement des langues. En effet, laisser la possibilité aux apprenants d'incarner un personnage dans un monde virtuel semble ouvrir les perspectives en ce qui concerne les activités en permettant de mettre en place des tâches telles qu'elles sont conçues dans la perspective actionnelle. De nombreuses études prenant pour sujet d'analyse World of Warcraft ont démontré la façon dont cet environnement proposait une écologie sémiotique riche et multimodale pour l'apprentissage des langues (Thorne \& Fischer, 2012; Zheng et alii, 2009). Ce jeu en ligne propose un environnement linguistique à même de développer chez ses utilisateurs une littératie complexe puisqu'elle amène ceux-ci à maîtriser plusieurs outils de communication du Web 2.0, afin de satisfaire à des besoins communicatifs variés 
au sein du jeu ou en dehors de celui-ci. Dans ce contexte informel, il a été constaté que l'inhibition à communiquer était inférieure (Peterson, 2010), du fait de l'absence contextuelle d'une instance évaluative en la personne, par exemple, du professeur. Autrement dit, le jeu vidéo, qu'il soit au départ à vocation pédagogique ou pas, peut permettre de mettre en place des interactions virtuellement situées propices à la réalisation de tâches qu'il n'est pas toujours possible de reproduire en classe.

Deux pratiques sont possibles (Alvarez \& Djaouti, 2012, p. 11) : soit l'enseignant détourne un jeu vidéo existant dans un but pédagogique, nous parlons alors de serious gaming, soit il utilise un jeu vidéo conçu spécifiquement avec une intention sérieuse et il s'agit alors d'un serious game. Ce dernier concept présente différentes catégories en fonction de l'intention (advergames pour les jeux publicitaires, Games with a purpose qui s'orientent vers la collecte et la production de données par les usagers, etc.); dans le cadre de cet article, nous nous intéressons aux learning games, les jeux vidéo qui ont pour but un apprentissage. Si l'utilisation du serious gaming présente l'avantage d'offrir aux apprenants une certaine authenticité dans le contexte, les tâches à effectuer et les interactions, il peut également s'écarter de manière trop importante des objectifs d'apprentissage, notamment linguistiques, de l'enseignant (Sykes, 2013, p.33). Au contraire, le jeu vidéo éducatif vise des objectifs, ainsi que des tâches, spécifiques et ciblés en fonction du niveau des apprenants. L'inconvénient se situe dans le fait que, si les ressorts ludiques sont trop peu importants ou inadaptés, le jeu aura peu de chances de déclencher chez le joueur une attitude ludique.

C'est ce dernier constat qui nous intéresse plus particulièrement et nous nous posons la question de savoir comment trouver un équilibre entre ludique et sérieux au moment de la conception d'un jeu vidéo éducatif. Dans l'exemple qui suit, nous nous limiterons donc à la question du serious game en présentant le cheminement et la réflexion que nous avons menés à travers la conception de plusieurs jeux vidéo pour l'apprentissage des langues.

\section{De la tension inhérente au jeu vidéo d'apprentissage : l'exemple d'Architecte 2015}

De la réflexion sur l'apport des jeux numériques par rapport aux jeux traditionnels en classe de langue ont émergé plusieurs projets de jeux pour l'apprentissage du FLE cherchant à exploiter les ressorts ludiques, techniques et scénaristiques des jeux vidéo auxquels nous avons participé. À travers ces différents projets est revenue constamment la question de la tension soulignée par Brougère (2012, p. 125) «entre logique du jeu ( gameplay) et logique de l'apprentissage", tension que nous présentons ci-dessous à travers l'historique de ces projets et que nous approfondirons avec le dernier d'entre eux afin de proposer des pistes de réflexion sur la façon d'appréhender la conception de jeux vidéo dédiés à l'apprentissage des langues.

D'après Brougère, la logique de conception d'un serious game incite à partir des objectifs d'apprentissage sur lesquels "on va greffer un gameplay plus ou moins adapté ». À l'opposé, le jeu de divertissement intègre « une logique ludique » « dans une histoire, un univers fictionnel adapté à [celle]-ci» (Brougère, 2012, p. 125). C'est ce que propose Djaouti (2011) avec son modèle de conception DICE pour Définir le contexte et le contenu sérieux (objectifs, moyens, contraintes), Imaginer un principe de jeu, Créer un prototype 
et Évaluer selon un processus itératif. Si nous n'avons pas suivi ce modèle pour le premier projet que nous présentons ci-dessous, nous avons tenté de l'appliquer aux deux derniers.

Thélème consiste en une maquette de jeu massivement multi-joueurs en ligne (MMOG) dont l'ambition est d'offrir aux internautes allophones un univers de cape et d'épée en français pour qu'ils profitent, tout en jouant, d'une immersion dans la langue-cible. Le principe de départ, même si le projet n'est pas allé plus loin que la maquette, propose, parallèlement aux quêtes, des missions plus adaptées au niveau linguistique des utilisateurs d'une autre langue. Le scénario et les évaluations prennent en compte les efforts linguistiques des joueurs qui interagissent à l'écrit par l'intermédiaire d'un chat. Les retours des apprenants et des enseignants après expérimentation sont en général positifs, mais mentionnent, pour les premiers, des difficultés à évoluer dans l'univers et à comprendre ce qu'ils doivent faire, pour les seconds, de n'avoir aucun contrôle sur ce que font leurs apprenants et de ne pas trouver leur place dans le concept. Ainsi, quand l'intention sérieuse n'apparait pas visiblement, notamment lorsque sont estompés les repères traditionnels de la classe - comme l'enseignant en position de domination ou des objectifs d'apprentissage clairement annoncés - le jeu vidéo à vocation pédagogique peut manquer sa cible. Comme si l'absence de ces repères familiers dans un jeu à vocation pédagogique empêchait les usagers d'entrer dans une attitude ludique. Mais cela reste à analyser plus profondément.

31 Dans le but d'apporter une réponse à ces observations, nous avons participé à la conception d'un nouveau modèle de jeu vidéo pour le FLE, centré sur le jeu d'aventure, mais beaucoup plus cadré que le précédent : Les Éonautes. Le concept reprend des idées de Thélème mais réduit certaines de ses fonctionnalités au profit d'un support plus adapté à la classe. Il s'agit d'un outil qui permet à l'enseignant de choisir sa progression didactique et de suivre les progrès des apprenants. Il peut effectuer des choix didactiques afin de créer un parcours d'apprentissage personnalisé pour chacun d'entre eux. Ces derniers ont moins de liberté d'action, car il ne s'agit plus d'un jeu en ligne où ils rencontrent d'autres joueurs ; ils ont par contre l'opportunité de découvrir différentes périodes culturellement marquées de la France à travers des voyages temporels durant lesquels ils interagissent à l'écrit avec des PNJ ou avec l'enseignant et les apprenants de la même classe par l'intermédiaire d'un chat privé. Les séquences sont limitées à 45 minutes de jeu en moyenne pour s'intégrer à l'heure de cours. Les missions sont davantage centrées sur un ou plusieurs objectifs linguistiques précis du niveau A2 ou B1 du Cadre européen commun de référence pour les langues et correspondent à des tâches telles que le préconise la perspective actionnelle. À l'inverse du premier projet, même si l'immersion visuelle et linguistique a été saluée, les retours sur Les Éonautes ont porté sur l'absence d'ouverture de l'espace de jeu et le caractère trop linéaire du parcours où, malgré la présence de ressorts ludiques, les utilisateurs ressentent trop fortement l'intention sérieuse.

Architecte 2015 est un jeu éducatif en 3D immersive, utilisant la reconnaissance de la parole, pour l'apprentissage de l'Allemand Langue Étrangère (Deutsch als Fremdsprache DAF) et du FLE. Ce projet sur trois ans reflète à travers son évolution cette tension que nous observons à travers les deux projets précédents et ce difficile équilibre à trouver entre ludique et sérieux. Le scénario du projet est destiné à des élèves de seconde (niveau A2 du Cadre européen commun de référence pour les langues, 2001). Le joueur doit accomplir un certain nombre d'actions dans le jeu afin d'aider une architecte à retrouver son père qui a disparu dans la maquette 3D de la Cathédrale de Strasbourg. Les consignes et les aides sont données en langue-cible et permettent de découvrir des éléments culturels sur 
ce monument, ainsi que le champ lexical de l'architecture attenant. L'apprenant, qui appartient à une génération ayant la faculté d'agir sur le monde virtuel, intègre le dispositif constitué d'une TV stéréoscopique 3-D, d'une paire de lunettes de stéréovision, d'une capture de mouvement à 6 degrés de liberté, d'un micro-casque et d'un smartphone et doit communiquer avec l'architecte pour passer un certain nombre d'épreuves. Les interactions avec le PNJ se font oralement, grâce au système de reconnaissance de la parole. Nous cherchons, à travers ce dispositif de réalité virtuelle, à provoquer chez le joueur des interactions non seulement verbales, mais aussi corporelles, autrement dit de l'engager dans une action qui nécessite également une interaction langagière. L'objectif attendu, sur le modèle de la perspective actionnelle, est de donner un sens à l'action, une signification qui serait celle du jeu et de l'univers dans lequel l'utilisateur est immergé de manière sensorielle, et par conséquent de donner du sens à l'utilisation de la langue-cible, tout en estompant la situation d'apprentissage.

Reprenant le principe du modèle DICE lors de la conception, nous avons sélectionné les objectifs communicatifs qui seraient présents dans le jeu (D), puis nous avons travaillé sur le scénario et la rédaction des dialogues (I) que nous avons implémentés dans le dispositif technique (C) et testés, dans sa version allemande, auprès de 46 élèves de seconde d'un lycée professionnel français en juin 2013 (E). ${ }^{4}$

L'observation nous a permis de constater que, non seulement les apprenants étaient statiques face à l'écran, alors même qu'ils avaient les moyens technologiques d'utiliser leur corps dans le jeu, mais qu'en plus, leur prise de parole était extrêmement réduite, se contentant de prononcer les mots-clés pour répondre au PNJ. Les feedbacks du PNJ étaient par ailleurs inadaptés, car, en cas d'incompréhension, celui-ci se contentait de répéter la même phrase avec un débit plus lent. Du reste, nous n'avions pas prévu que les apprenants puissent poser des questions sur l'environnement ou plus simplement sur le sens des mots.

À la question "Comment tu appellerais ce que tu viens de faire? ", les entretiens ont fait ressortir que $41,5 \%$ des élèves considéraient l'expérience comme un «jeu» ou un «jeu pédagogique » contre $22,5 \%$ pour "un exercice »; les $36 \%$ restant la qualifiant de « simulation » ou d'« immersion ». Les apprenants considérant Architecte 2015 comme un jeu le justifient en utilisant le mot « ludique». Nous avons cependant noté une tendance très forte à utiliser ce qualificatif par rapport au dispositif lui-même et ce qu'il permet, à savoir l'immersion visuelle, la navigation et surtout les interactions avec les objets et le décor, et non pas par rapport au scénario. Au contraire, les remarques négatives portent davantage sur les dialogues, d'une part les difficultés face à la compréhension de la langue, d'autre part les prises de parole trop longues du PNJ, les consignes qui manquent de clarté et la répétition des actions et des consignes. Nous avons conclu que le produit en l'état correspondait plus à une simulation qu'à un jeu vidéo et qu'il fallait complètement revoir notre façon d'appréhender la conception d'un jeu à destination de l'enseignement des langues. Pour citer un élève, « en gros, c'était fun de faire les choses, mais ça servait quasiment à rien, quoi. C'était juste pour un peu nous distraire en même temps qu'on parlait allemand, quoi ».

En revenant sur nos choix didactiques et scénaristiques, nous avons constaté que partir de la définition des objectifs d'apprentissage avait eu pour inconvénient de nous faire entrer dans une logique de contrôle de la prise de parole des utilisateurs. À partir de là s'est naturellement mis en place un scénario séquencé, durant lequel le joueur n'a pas d'autre choix que de valider un verrou scénaristique pour passer à un autre, et les 
dialogues ont pris la forme d'une liste chaînée avec conditions (Koster, 2005, p. 42), enfermant ainsi l'utilisateur dans une boucle où il n'est jamais à l'origine de l'interaction et où il ne peut que faire la bonne action ou donner la bonne réponse pour en sortir. Avec cette approche, nous mettons le joueur dans une position passive qui se contente de suivre des consignes et n'a aucun pouvoir de décision, critère pourtant fondamental d'après notre positionnement de départ sur les caractéristiques du jeu.

En effet, certains retours des élèves pendant le test confirment que ce critère a de l'importance pour qualifier une expérience de ludique. Un lycéen, quand il lui est demandé pourquoi il considère l'expérience comme un exercice, répond: «parce qu'on est guidé, parce que par exemple dans un jeu vidéo, on fait euh, certes on a des missions ou ce genre de choses, mais là on avait vraiment quelque chose qui nous était dicté et qu'on devait faire». Nous pouvons alors nous demander, une fois de plus, quelle est la valeur ajoutée d'un jeu de ce type, qui se veut pourtant ouvert et interactif, par rapport à des jeux traditionnels transposés sur écran.

Forts de ces constats, nous avons complètement revu notre modèle de conception avec pour but d'introduire davantage de ressorts ludiques et surtout laisser plus de liberté d'action au joueur. Nous avons alors fait le choix de partir non plus des objectifs, mais du scénario et des actions à y faire et de terminer par la définition des objectifs langagiers nécessaires pour pouvoir réussir le jeu et les tâches. La conséquence sur la structure du scénario est que les verrous scénaristiques ne sont plus séquencés, mais imbriqués. Autrement dit, les étapes par lesquelles le joueur doit passer dans un ordre prédéfini sont limitées, au profit d'une plus grande liberté d'action : l'utilisateur peut en grande partie décider de l'ordre dans lequel il va accomplir les épreuves ou les énigmes. Pour provoquer davantage d'interactions corporelles, nous avons introduit des épreuves qui amènent le joueur à se déplacer dans le monde virtuel, comme des objets cachés à retrouver ou la présence d'un opposant offensif qu'il faut éviter.

Pour stimuler la prise de parole, nous avons là aussi décidé d'inverser le modèle. Plutôt que de partir d'une consigne ou d'une question formulée par le PNJ, nous nous sommes demandé quels événements le joueur est susceptible de déclencher. Nous avons déterminé qu'il pouvait, entre autres, ne rien faire, entrer et sortir dans des zones prédéterminées, désigner un objet sur la scène, accomplir une action sans prendre la parole, prendre la parole pour dire son incompréhension, poser une question, répondre ou commenter l'action. Pour chaque événement, relié à une zone ou pas, nous avons ensuite rédigé les feedbacks du PNJ. La structure du dialogue a pris la forme d'un arbre à conditions qui propose des embranchements multiples avec des états parallèles (Koster, 2005, p. 44). De ce fait, le joueur est la plupart du temps à l'origine de l'interaction et donc plus libre. Il lui faut demander ce qu'il faut faire, mais il peut éventuellement aussi traverser le jeu en n'ayant que très peu pris la parole, en développant d'autres stratégies comme comprendre ce qu'il faut faire grâce au contexte ou en agissant plutôt qu'en parlant. Et c'est bien là une des caractéristiques mises en avant par la perspective actionnelle. Nous avons cependant introduit un bonus de parole, en plus du bonus qui valide la réussite de l'action, afin de l'inciter à s'exprimer dans la langue-cible. Cette nouvelle version est désormais finalisée et fera l'objet d'un nouveau test fin 2014. 


\section{Conclusion}

40

Ainsi, pour que le jeu numérique apporte un intérêt supplémentaire à l'apprentissage des langues par rapport au jeu traditionnel, nous ne pouvons pas nous contenter de reproduire de l'ancien pour faire du nouveau. Des expérimentations, des essais et des erreurs sont nécessaires pour apporter à l'enseignement une réflexion, une pratique et des outils valides. Le plus difficile est, que ce soit pour le concepteur, pour l'enseignant ou l'apprenant, de dépasser ses propres représentations et une pensée héritée aussi bien des méthodologies anciennes qui ignorent le jeu que des pratiques plus récentes qui ont une conception trop fermée du jeu.

41

En effet, le jeu en classe de langue prend avant tout la forme d'un support ou d'une activité complémentaire permettant d'introduire un peu de variété et d'originalité parmi les autres exercices d'entraînement linguistique. Cette pratique médiatisée par les technologies numériques met encore plus en avant cette instrumentation fermée du jeu et la difficulté à sortir des cadres. Pour profiter des potentialités du jeu numérique, l'enseignant doit consacrer du temps à ce dernier, notamment en se formant à ses usages, mais aussi en réfléchissant à la façon de l'intégrer dans la classe de langue. Ce qui implique également de modifier sa position pédagogique et accepter de ne pas tout contrôler. Car pour que le jeu soit propice au déclenchement d'une attitude ludique, il doit, entre autres, être ouvert et laisser la possibilité à l'apprenant de prendre ses propres décisions, décisions qui peuvent aller à l'encontre de ce qu'attendait l'enseignant.

\section{BIBLIOGRAPHIE}

ABRY D., FERT C., PARPETTE C. \& STAUBER J. (2007a), Ici 1. Guide pédagogique, Paris, CLE International.

ABRY D., FERT C., PARPETTE C., STAUBER J. et al. (2007b), Ici 1. Méthode de français, Paris, CLE International.

ALVAREZ J. (2007), Du jeu vidéo au Serious game. Approches culturelle, pragmatique et formelle, Thèse de Doctorat en Science de la communication et de l'information, Université de Toulouse II et III.

ALVAREZ J. \& DJAOUTI D. (2012), Introduction au Serious game, Paris, Questions théoriques.

BAGLIETO D., GIRARDEAU B. \& MISTICHELLI M. (2011a), Agenda 1. Guide pédagogique, Paris, Hachette Français langue étrangère.

BAGLIETO D., GIRARDEAU B. \& MISTICHELLI M. (2011b), Agenda 1. Méthode de français, Paris, Hachette Français langue étrangère.

BROUGÈRE G. (2005), Jouer / Apprendre, Paris, Economica, Coll. Éducation.

BROUGÈRE G. (2012), « Le jeu peut-il être sérieux ? Revisiter Jouer / Apprendre en temps de serious game ", Australian Journal of French Studies, vol XLIX, n² 2, pp. 117-129. 
CAPELLE G. \& MENAND R. (2009a), Le nouveau Taxi 1. Méthode de Français, Paris, Hachette Français langue étrangère.

CAPELLE G. \& MENAND R. (2009b), Le nouveau Taxi 1. Méthode de Français. Guide pédagogique, Paris, Hachette Français langue étrangère.

CARÉ F. \& DEBYSER J.-M. (1978), Jeu, langage et créativité. Les jeux dans la classe de français, Paris, Hachette FLE.

Conseil de l'Europe (2001), Un cadre européen commun de référence pour les langues : apprendre, enseigner, évaluer, Strasbourg, Division des Politiques Linguistiques.

DETERDING S., DIXON D., KHALED R. \& NACKE L. (2011), « From game design elements to gamefulness : defining " gamification » ", MindTrek '11: Proceedings of the 15th International Academic MindTrek Conference : Envisioning Future Media Environments, New York, ACM, pp. 9-15.

DE VALLANGE (1730), Art d'ensègner le latin aux petits enfans en les divertissant et sans qu'ils s'en aperçoivent, Paris, A. Gandouin, Laisnel, Veuve P. Ribout.

DJAOUTI D. (2011), Serious Game Design. Considérations théoriques et techniques sur la création de jeux vidéo à vocation utilitaire, Thèse de Doctorat en Informatique, Université de Toulouse III.

DUBOIS A.-L. \& LEROLLE M. (2008), Scénario 1. Méthode de français, Paris, Hachette Français langue étrangère.

ELLIS R. (2003), Task-based Language Learning and Teaching, Oxford : Oxford University Press.

FLUMIAN C., LABASCOULE J., LAUSE C. \& ROYER C. (2011), Nouveau Rond-point 1. Méthode de français base sur l'apprentissage par les tâches, Paris, Éditions Maison des langues.

GENVO S. \& POIX J. (2003), « Concevoir un jeu vidéo éducatif », Les dossiers de l'ingénierie éducative, $\mathrm{n}^{\circ} 44$, pp. $77-79$.

GIRARDET J. \& GIBBE C. (2010a), Echo A1. Méthode de français. Livre du professeur, Paris, CLE International.

GIRARDET J. \& PÉCHEUR J. (2010b), Echo A1. Méthode de français. Méthode de français, Paris, CLE International.

HENRIOT J. (1983), Le jeu [1969], Paris, Synonyme.

HUGOT C., KIZIRIAN V. M., WAENDENDRIES M., BERTHET A. \& DAILL E. (2012), Alter Ego + A1. Méthode de français, Paris, Hachette Français langue étrangère.

HYMES D. H. (1984), Vers la compétence de communication, Paris, Hatier.

KOSTER R. (2005), A grammar of gameplay - Game atoms : can games be diagrammed ?. [En ligne] Consulté le 04/03/2014 : http://www.raphkoster.com/gaming/atof/ grammarofgameplay.pdf.

MAUCO O. (2012), «Sur la gamification », Gameinsociety. [En ligne] consulté le 17 mai 2015 : http://www.gameinsociety.com/public/Sur_la_gamification_-_Mauco__gameinsociety.pdf MÉRIEUX R. \& LOISEAU Y. (2004a), Connexions, Niveau 1. Guide pédagogique, Paris, Didier.

MÉRIEUX R., LOISEAU Y. (2004b), Connexions, Niveau 1. Méthode de français, Paris, Didier.

MÉRIEUX R., LOISEAU Y. (2008), Latitudes 1. Méthode de français, Paris, Didier.

MONDADA L., PEKAREK DOEHLER S. (2000), « Interaction sociale et cognition située : quels modèles pour la recherche sur l'acquisition des langues ? ", Acquisition et interaction en langue étrangère, $\mathrm{n}^{\circ} 12$. 
MONNERIE-GOARIN A., SCHMITT S., SAINTENOY S., SZARVAS B. (2006a), Métro Saint-Michel 1. Méthode de français, Paris, CLE International.

MONNERIE-GOARIN A., SCHMITT S., SAINTENOY S., SIRÉJOLS E., SZARVAS, B. (2006b), Métro SaintMichel 1. Méthode de français. Livre du professeur, Paris, CLE International.

OLLIVIER C. (2012), « Approche interactionnelle et didactique invisible - Deux concepts pour la conception et la mise en œuvre de tâches sur le web social ", Médias sociaux et apprentissage des langues : (r)évolution? Alsic, vol 15, $\mathrm{n}^{\circ} 1$.

PETERSON M. (2010), « Computerized games and simulations in computer-assisted language learning : A meta-analysis of research », Simulation \& Gaming, vol. 41, n 1, New York, Sage Publications, pp. 72-93.

PUREN C. (2012), Histoire des méthodologies de l'enseignement des langues [1988], Paris : Nathan.

ROBERT P., REY-DEBOVE J. \& REY A. (2012), Le petit Robert : Dictionnaire alphabétique et analogique de la langue française, Paris, Le Robert.

ROY M. (2014), « L'analyse qualitative de contenu d'entretien : un outil pour appréhender le sentiment de présence en environnement virtuel », Eurographics 2014, Communication dans le cadre du Workshop « Immersive Learning and Education », 7 avril 2014, Strasbourg.

SAUVÉ L. \& KAUFMAN D. (dir.). (2010), Jeux et simulations éducatifs. Études de cas et leçons apprises, Québec, Presses de l'Université du Québec.

SCHMOLL L. \& KOECHER L. (2006), « Internet et l'enseignement/apprentissage du Français Langue Étrangère : une exploitation limitée de ses spécificités », Dialogues et Cultures, $\mathrm{n}^{\circ} 53$, pp. 587-595.

SCHMOLL L. (2011), « Usages éducatifs des jeux en ligne : l'exemple de l'apprentissage des langues ", Revue des sciences sociales, $\mathrm{n}^{\circ} 45$, pp. 148-157.

SILVA H. (2008), Le jeu en classe de langue, Paris, CLE International.

SYKES J. M. (2013), « "Just” playing games ? A look at the digital games for language learning », The Language Educator, pp. 32-35.

THORNE S. L. \& FISCHER I. (2012), « Online gaming as sociable media », Alsic, vol. 15, nº 1.

Verbi Software (2014), MAXQDA für Windows. Referenzhandbuch. [En ligne] consulté le 14/10/2014 : http://www.maxqda.de/download/manuals/MAX11_manual_ger.pdf.

WEISS, F. (2002), Jouer, communiquer, apprendre, Coll. Pratiques de classe, Hachette FLE, Paris.

ZHENG D., YOUNG M., WAGNER M. \& BREWER R. (2009), « Negotiation for action : English language learning in game-based virtual worlds ", The Modern Language Journal, n 93, pp. 489511.

\section{ANNEXES}

\section{Liste des jeux numériques cités}

Architecte 2015. Dernière consultation le 14/10/2014 : http://www.eveil-3d.eu/francais/ index.php. 
Le projet EVEIL-3D est cofinancé par l'Union Européenne via le Fonds Européen de Développement Régional (FEDER), dans le cadre du programme INTERREG IV - Rhin Supérieur.

Babelweb. Dernière consultation le 14/10/2014 : http://www.babel-web.eu.

Site réalisé dans le cadre des projets européens Babelweb (135610-LLP-1-2007-1-AT-KA2KA2MP) et Babelweb.Pro (519258-LLP-1-2011-1-AT-KA4-KA4MP) financés avec le soutien de la Commission européenne.

Jeu de l'oie. Apprendre le français en s'amusant. Dernière consultation le 14/10/2014 : http:// jeudeloie.free.fr/.

Les Éonautes. Dernière consultation le 14/10/2014 : http://www.eonautes.com/fr/ eonautes-serious-game-fle.html.

Jeu réalisé par Almédia, avec le soutien du Ministère de l'Éducation nationale.

Thélème. Dernière consultation le 14/10/2014 : http://www.theleme-lejeu.com/.

Jeu réalisé par Almédia et le Laboratoire Cultures et sociétés en Europe (devenu depuis Dynamiques européennes - UMR7367) grâce au fonds de maturation de projets innovants Conectus.

\section{NOTES}

1. Expression utilisée de façon consensuelle par les acteurs de l'enseignement du FLE pour désigner les mineurs d'une tranche d'âge allant vraisemblablement de 15 à 18 ou 21 ans.

2. L'approche communicative a pour ambition d'amener l'apprenant à pouvoir interagir dans des situations de communication définies. L'enseignement insiste sur le sens et le contexte et non pas sur les formes et les structures de la langue. Le jeu de rôles devient l'activité de production orale principale.

3. Propos recueillis lors d'une expérimentation pour le projet Architecte 2015 (voir plus loin).

4. Les lycéens sont enregistrés pendant le test (enregistrement audio pour les dialogues, enregistrement vidéo pour les attitudes). L'expérience est suivie d'un entretien individuel enregistré (audio uniquement) pour évaluer notamment leurs impressions sur le dispositif, le scénario et l'intérêt d'un jeu pour l'allemand. L'observation en direct est faite à l'aide d'une grille critériée, prenant en compte les différents types de mouvement, les réactions verbales et nonverbales, les sollicitations vers les intervenants et les types de prise de parole dans le jeu. Les entretiens, correspondant à neuf heures et cinquante-sept minutes d'enregistrement, sont transcrits et analysés à l'aide du logiciel MAXQDA (Roy, 2014).

\section{RÉSUMÉS}

Cet article interroge la place du jeu dans l'enseignement des langues et s'intéresse plus précisément aux façons dont la transition entre l'utilisation des jeux dits traditionnels en classe et leur adaptation sur supports multimédia s'est effectuée. Les jeux sont-ils les mêmes ou ont-ils 
changé ? Le passage au numérique a-t-il permis de préserver les ressorts ludiques spécifiques à ces jeux ou les a-t-il modifiés au profit de l'intention et du contenu sérieux? Nous questionnerons ainsi l'apport que le numérique peut éventuellement offrir au jeu traditionnel à la lumière des méthodologies d'enseignement-apprentissage actuelles. Pour aller plus loin, nous nous demanderons comment penser la conception d'un jeu vidéo éducatif en respectant, d'une part, un certain équilibre entre ludique et sérieux et d'autre part, la démarche didactique qui a présidé aux choix scénaristiques. Pour ce faire, nous prendrons comme exemple la conception d'un jeu d'apprentissage en immersion 3D stéréoscopique pour l'apprentissage du français et de l'allemand.

This article questions the place of games in language teaching and more specifically examines how the transition between the use of traditional games in the classroom and their adaptation to digital media has been carried out. Are these games the same or have they changed? Has the digital switchover preserved the entertaining aspects of these games or have these aspects changed in order to accommodate more serious content? We will thus examine the potential added value of digital versus traditional games in the light of current teaching methodologies and we will discuss the design of learning games with regard to striking an adequate balance between fun and serious as well as respecting the didactic guidelines of the game. By way of an example we will refer to the design of a 3D immersive learning game for French and German languages.

\section{INDEX}

Mots-clés : jeu vidéo d'apprentissage, apprentissage des langues, tâches, perspective actionnelle, attitude ludique

Keywords : learning games, language learning, tasks, action-based learning, playful attitude

\section{AUTEUR}

\section{LAURENCE SCHMOLL}

Université de Strasbourg 